\title{
ON THE DESIGN OF REAL AND COMPLEX FIR FILTERS WITH FLATNESS AND PEAK ERROR CONSTRAINTS Using Semidefinite Programming
}

\author{
S. C. Chan and K. M. Tsui
}

Department of Electrical and Electronic Engineering,

The University of Hong Kong, Pokfulam Road, Hong Kong.

\begin{abstract}
This paper studies the problem of designing digital finite duration impulse response (FIR) filters with prescribed flatness and peak error constraints using semidefinite programming (SDP). SDP is a powerful convex optimization method, where linear and convex quadratic inequality constraints can readily be incorporated. This property is utilized for the optimal minimax and least squares (LS) design of linear-phase and low-delay FIR filters with prescribed magnitude flatness and peak design error, which are formulated as a set of linear equality and convex quadratic inequality constraints, respectively. A method for structurally imposing these equality constraints in the SDP formulation is also proposed. Using these results, the design approach is further extended to the design of constrained complex coefficient FIR filters and variable digital filters (VDFs). Design examples are given to demonstrate the effectiveness of the approach.
\end{abstract}

\section{INTRODUCTION}

Recently, SDP has been proposed as a versatile framework for designing digital FIR and IIR filters [1-6]. It was found that many filter design problems can be cast as SDP problem. Another advantage of SDP which we shall utilize in this paper is its ability to satisfy multiple objectives expressed in terms of a set of linear and convex quadratic constraints. In fact, the pioneer works in $[1,2]$ formulates the minimax and the least squares design criteria as a set of such inequalities. This motivates us to study the design of digital FIR filters with more general constraints such as magnitude flatness (such as multiple zeros in magnitude response) and peak design error constraints. Interested readers are referred to [7] for more information of SDP, and its application to the design of FIR and IIR filters [16]. For linear-phase (LP) FIR filters, linear programming has been proposed [8] as a general framework for handling the additional linear equality and inequality constraints. Since SDP is an extension of linear programming, the SDP-based constrained FIR filter design method studied in this paper can be viewed as its generalization to handle convex quadratic objective function and constraints, which allows optima minimax (and least squares) passband linear-phase FIR filters subject to linear equalities and convex quadratic inequalities to be designed. There are previous attempts in incorporating linear equality constraints in least squares design of FIR filters $[9,10]$. The design problem is usually formulated as a quadratic programming problem with linearly equality constraints (QPLC), generally known as the eigenfilter design method which can be extended to the complex FIR filter design [11,12]. Advantages of these approaches are their good performance and low design complexities. On the other hand, the SDP approach is capable of handling more general types of quadratic constraints and design criterion. Another interesting property of SDP is that whenever a design problem can be formulated as SDP, it means that the problem is convex and the optimality of the solution, if there is any, is guaranteed.

In this paper, we mainly focus on the FIR filter design problem with prescribed flatness and peak error constraints [13]. Constraints like magnitude flatness and multiple zeros are desirable in designing sample rate converter in order to suppress the alias components, and the design of wavelet basis. On the other hand, peak error constraints are useful to limit the sidelobe and undesirable peaks in filters with wide unconstrained transition band. Both least squares and minimax criterion will be considered, though SDP is particular attractive for the latter. The magnitude flatness constraints are derived through a simple relation between the derivatives of the filter response and its ideal counterpart. This yields a set of linear equality constraints, where some of the redundant variables can be expressed in terms of the remaining variables, and are eliminated. As a result, the equality constraints can be structurally imposed in the SDP formulation, leading to fewer numbers of variables. Within the SDP framework, these linear equality and convex quadratic inequalities constraints such as peak design error can be integrated together in the optimal design of real and complex FIR filters with minimax and LS errors satisfying these constraints. This offers an improved tradeoff between magnitude flatness, and passband and stopband ripples over conventional linear-phase maximally-flat filter design methods, say Hilbert Transformers [14], as we shall demonstrate in the design example section. In addition, since the FIR filters are not limited to linear-phase, the system delay can further be reduced. The new design method also offers more freedom and higher performance over tradition LS method in designing VDFs [15] with either minimax or LS design criteria, and a wide variety of constraints including magnitude flatness and peak design error constraints. Design results show that the SDP method offers an attractive alternative to traditional design methods because of its optimality, generality and flexibility.

The paper is organized as follows: Section II is devoted to the SDP formulation of real and complex FIR filters, as well as VDFs design, based on either minimax or LS criteria. Methods for deriving the magnitude flatness/zero and peak design error constraints will be introduced in section III. Design examples are given in Section IV to demonstrate the effectiveness of the proposed approach. Finally, conclusion is drawn in Section V.

\section{SDP FORMULATION}

\section{A. - Real-valued FIR filter design}

Denote the transfer function of the FIR filter to be designed by

$$
H(z)=\sum_{n=0}^{N-1} h(n) z^{-n},
$$

where $h(n)$ is the impulse response of the filter and $N$ is the filter length. We want to approximate the desired response $H_{d}(\omega)$ by $H(z)$ in the minimax sense. That is:

$$
\min _{h(n)} \max W(\omega)\left|H\left(e^{j \omega}\right)-H_{d}(\omega)\right|, \quad \omega \in \Omega_{I},
$$

where $W(\omega)$ is a positive weighting function, and $\Omega_{I} \in[-\pi, \pi]$ is the frequency interval of interest. The frequency response $H\left(e^{j \omega}\right)$ can be written as:

$$
\begin{aligned}
& \qquad \begin{aligned}
H\left(e^{j \omega}\right) & =\sum_{n=0}^{N-1} h(n) e^{-j n \omega}=\boldsymbol{h}^{T}\{\boldsymbol{c}(\omega)-j \boldsymbol{s}(\omega)\}, \\
\boldsymbol{h} & =[h(0), \ldots, h(N-1)]^{T}, \\
\text { where } \quad \boldsymbol{c}(\omega) & =[1, \cos \omega \ldots, \cos ((N-1) \omega)]^{T} \text { and } \\
\boldsymbol{s}(\omega) & =[0, \sin \omega \ldots, \sin ((N-1) \omega)]^{T} .
\end{aligned}
\end{aligned}
$$

The minimization problem in (2-2) can be reformulated as:

$$
\min _{h} \delta \quad \text { subject to } W^{2}(\omega)\left|H\left(e^{j \omega}\right)-H_{d}(\omega)\right|^{2} \leq \delta \text {. (2-5) }
$$

Let $H_{R_{-} d}(\omega)$ and $H_{I_{-} d}(\omega)$ be the real and imaginary parts of $H_{d}(\omega),(2-5)$ can be written as: 


$$
\begin{aligned}
& \min _{h} \delta \quad \text { subject to } \delta-\left\{\alpha_{R}^{2}(\omega)+\alpha_{I}^{2}(\omega)\right\} \geq 0, \\
& \text { where } \begin{array}{l}
\alpha_{R}(\omega)=W(\omega) \cdot\left|\boldsymbol{h}^{T} \boldsymbol{c}(\omega)-H_{R_{-} d}(\omega)\right| \text { and } \\
\alpha_{I}(\omega)=W(\omega) \cdot\left|\boldsymbol{h}^{T} \boldsymbol{s}(\omega)+H_{R_{-} I}(\omega)\right| .
\end{array}
\end{aligned}
$$

Using Schur complement [2,7], the constraints in (2-6) is equivalent to:

$$
\boldsymbol{\Gamma}(\boldsymbol{h}, \omega)=\left[\begin{array}{ccc}
\delta & \alpha_{R}(\omega) & \alpha_{I}(\omega) \\
\alpha_{R}(\omega) & 1 & 0 \\
\alpha_{I}(\omega) & 0 & 1
\end{array}\right] \succeq 0 .
$$

Digitizing the frequency variable $\omega$ over a dense set of frequencies $\left\{\omega_{i}, 1 \leq i \leq m\right\}$ on the frequency of interests, the constraints in (2-8) becomes:

$\boldsymbol{\Gamma}\left(\boldsymbol{h}, \omega_{i}\right) \succeq 0,\left\{\omega_{i}, 1 \leq i \leq m\right\}$.

It can be shown that (2-9) can be stacked together to form the following set of linear matrix inequalities (LMIs):

$\boldsymbol{\Gamma}(\boldsymbol{h}) \succeq 0$,

where $\boldsymbol{\Gamma}(\boldsymbol{h})=\operatorname{diag}\left\{\boldsymbol{\Gamma}\left(\boldsymbol{h}, \omega_{1}\right), \ldots, \boldsymbol{\Gamma}\left(\boldsymbol{h}, \omega_{m}\right)\right\}$. Furthermore, by defining the augmented variable $\boldsymbol{x}=\left[\begin{array}{ll}\delta & \boldsymbol{h}^{T}\end{array}\right]^{T},(2-6)$ can be cast to the standard SDP problem as follows [2]:

$$
\min _{\boldsymbol{x}} \boldsymbol{c}^{T} \boldsymbol{x} \text { subject to } \boldsymbol{\Gamma}(\boldsymbol{x}) \succeq 0,
$$

where $c=[1,0, \ldots .0]^{T}$. Alternatively, the least square stopband attenuation can be minimized:

$$
E_{L S}(\boldsymbol{h})=\int_{\Omega_{I}} W(\omega)\left|H\left(e^{j \omega}\right)-H_{d}(\omega)\right|^{2} d \omega,
$$

This is a quadratic programming problem:

$$
\begin{aligned}
\min _{\boldsymbol{h}} & \boldsymbol{h}^{T} \boldsymbol{Q h}+\boldsymbol{h}^{T} \boldsymbol{g}+k, \\
& \boldsymbol{Q}=\oint_{\Omega_{I}} W(\omega) \cdot\left[\boldsymbol{c}(\omega) \boldsymbol{c}(\omega)^{T}+\boldsymbol{s}(\omega) \boldsymbol{s}(\omega)^{T}\right] d \omega, \\
\text { where } \quad & \boldsymbol{g}=\oint_{\Omega_{I}} W(\omega)\left[\boldsymbol{c}(\omega) H_{R_{-} d}(\omega)+\boldsymbol{s}(\omega) H_{I_{-} d}(\omega)\right] d \omega \\
& \text { and } k=\oint_{\Omega_{I}} W(\omega)\left|H_{d}(\omega)\right|^{2} d \omega .
\end{aligned}
$$

To formulate (2-13) as a SDP problem, decompose $\boldsymbol{Q}$ as $\boldsymbol{Q}=\boldsymbol{P}^{T} \boldsymbol{P}$, one can reformulate it by means of Schur complement, as

$$
\min _{\boldsymbol{x}} \quad \boldsymbol{c}^{T} \boldsymbol{x} \quad \text { subject to }\left[\begin{array}{cc}
\delta-\boldsymbol{h}^{T} \boldsymbol{g}-k & \boldsymbol{h}^{T} \boldsymbol{P}^{T} \\
\boldsymbol{P h} & 1
\end{array}\right] \succeq 0 .(2-14)
$$

The advantage of formulating the objective function as a set of LMIs is that the resulting problem is convex and the optimal solution, if it exists, can be found. In addition, other linear equalities or convex quadratic constraints can easily be incorporated, as we shall illustrate in later sections.

\section{B. - Complex-valued FIR filter design}

Suppose that the impulse response of the complex FIR filter is given by $\boldsymbol{h}=\boldsymbol{h}_{R}+j \boldsymbol{h}_{I}$, where $\boldsymbol{h}_{R}=\left[h_{R}(0), \ldots, h_{R}(N-1)\right]^{T}$ and $\boldsymbol{h}_{I}=\left[h_{I}(0), \ldots, h_{I}(N-1)\right]^{T}$. If we define that:

$$
\begin{aligned}
& \boldsymbol{a}=\left[\boldsymbol{h}_{R}^{T}, \boldsymbol{h}_{I}^{T}\right]^{T}, \boldsymbol{c}_{1}(\omega)=\left[\boldsymbol{c}(\omega)^{T}, \boldsymbol{s}(\omega)^{T}\right]^{T} \text { and } \\
& \boldsymbol{c}_{2}(\omega)=\left[\boldsymbol{s}(\omega)^{T},-\boldsymbol{c}(\omega)^{T}\right]^{T},
\end{aligned}
$$

then (2-3) can be written as:

$$
H\left(e^{j \omega}\right)=\boldsymbol{a}^{T}\left\{\boldsymbol{c}_{1}(\omega)-j \boldsymbol{c}_{2}(\omega)\right\}
$$

By replacing $\boldsymbol{h}, \boldsymbol{c}(\omega)$ and $\boldsymbol{s}(\omega)$, as defined in (2-4), with $\boldsymbol{a}$, $c_{1}(\omega)$ and $c_{2}(\omega)$, respectively, the minimization problems in (2-2) and (2-12) for complex FIR filters can be solved by SDP.

\section{C. - Real-valued variable digital filter (VDF) design}

In a VDF [15], the desired response $H_{d}(\omega, \phi)$ is a function of a spectral parameter $\phi$ (also known as tuning or control parameters). The spectral characteristics of a VDF can therefore be continuously varied by changing $\phi$. The impulse response of the VDF under consideration is assumed to be a linear combination of a polynomial basis function of the spectral parameter $\phi$ and subfilter coefficients $h_{l}(n)$, and it is given by:

$$
h(n, \phi)=\sum_{l=0}^{L-1} h_{l}(n) \cdot \phi^{l},
$$

where $\phi$ is assumed to vary linearly over a finite interval. The z-transform of the polynomial-based VDF is then given by:

$$
H(z, \phi)=\sum_{n=0}^{N-1 L-1} \sum_{l=0} h_{l}(n) \cdot \phi^{l} z^{-n} .
$$

To simply the notation, substitute $m=n+N l$ and $z=e^{j \omega}$ into (2-18), one gets

$$
H\left(e^{j \omega}, \phi\right)=\sum_{m=0}^{L N-1} a_{m} \phi^{l} e^{-j \omega n},
$$

where $n=\bmod (m, N)$ and $l=(m-n) / N$. (2-19) can be rewritten more compactly in matrix form as follows:

$$
\begin{aligned}
& H\left(e^{j \omega}, \phi\right)=\boldsymbol{a}^{T}\{\boldsymbol{c}(\omega, \phi)-j \boldsymbol{s}(\omega, \phi)\}, \\
& \boldsymbol{a}=\left[a_{0}, \ldots, a_{L N-1}\right]^{T}=\left[\boldsymbol{h}_{\boldsymbol{0}}{ }^{T}, \ldots, \boldsymbol{h}_{L-1}{ }^{T}\right]^{T}, \\
& \text { where } \quad \boldsymbol{h}_{l}=\left[h_{l}(0), \ldots, h_{l}(N-1)\right]^{T} \text { for } l=0,1, \ldots, L-1 \text {, } \\
& \boldsymbol{c}(\omega, \phi)=\left[\boldsymbol{c}(\omega)^{T}, \phi^{1} \cdot \boldsymbol{c}(\omega)^{T}, \ldots, \phi^{L-1} \cdot \boldsymbol{c}(\omega)^{T}\right]^{T}, \\
& \boldsymbol{s}(\omega, \phi)=\left[\boldsymbol{s}(\omega)^{T}, \phi^{1} \cdot \boldsymbol{s}(\omega)^{T}, \ldots, \phi^{L-1} \cdot \boldsymbol{s}(\omega)^{T}\right]^{T} .
\end{aligned}
$$

Again, in order to design VDF with either minimax or LS criteria, we replace $\boldsymbol{h}, \boldsymbol{c}(\omega)$ and $\boldsymbol{s}(\omega)$ with $\boldsymbol{a}, \boldsymbol{c}(\omega, \phi)$ and $\boldsymbol{s}(\omega, \phi)$, respectively, and digitize both $\omega$ and $\phi$ densely. The generalization to complex-valued VDF is similar. Details are omitted due to page limitation.

\section{Constrained Filter Design USIng SDP}

\section{A. - Imposing linear equality constraints}

As mentioned earlier, it is often required to impose certain constraints on the frequency characteristics when designing digital filters. One commonly encountered constraint is the linear equality constraints, which includes magnitude flatness at certain frequency points in the passband. Constraints such as a prescribed number of zeros at the stopband also belong to this category. To incorporate these constraints into SDP, the following relation between the derivatives of the design response and its ideal counterparts is employed:

$$
\left.\frac{d^{i}}{d z^{i}} H(\omega)\right|_{\omega=\hat{\omega}}=\left.\frac{d^{i}}{d z^{i}} H_{d}(\omega)\right|_{\omega=\hat{\omega}}, i=0,1, \ldots, K-1
$$

(3-1) tells us that the filter to be designed, $H(\omega)$, should approximate the desired response at $\omega=\hat{\omega}$ up to the $(K-1)$-th derivatives. We now give two simple examples.

i) Magnitude flatness constraint at the passband:

Suppose that the desired passband response is of the form $e^{-j \omega \tau+\phi}$ for any $\phi \in[-\pi, \pi]$, where $\tau=(N-1) / 2-D$ is the group delay; and $D$ is the delay reduction parameter. To impose a magnitude flatness of order $U_{\hat{\omega}_{p}}-1$ on $H(z)$ at $\hat{\omega}_{p} \in \Omega_{p}$, the passband, we have:

$$
\sum_{n=1}^{N-1}(n-\tau)^{u} \cdot h(n) \cdot e^{-j \hat{\omega}_{p}(n-\tau)}=\left\{\begin{array}{ll}
e^{\phi}, & u=0 \\
0, & u=1, \ldots, U_{\hat{\omega}_{p}}-1
\end{array},\right.
$$

and its matrix form is given by:

$$
\boldsymbol{U}_{\hat{\omega}_{p}} \cdot \boldsymbol{h}=\boldsymbol{d}_{\hat{\omega}_{p}}, u=0,1, \ldots, U_{\hat{\omega}_{p}}-1 .
$$

where $\left[\mathbf{U}_{\hat{\omega}_{p}}\right]_{u, n}=(n-\tau)^{u} e^{-j \hat{\omega}_{p}(n-\tau)}$ and $\boldsymbol{d}_{\hat{\omega}_{p}}=\left[e^{\phi}, 0, \ldots, 0\right]^{T}$. The constraints in (3-1) can also be applied to other form of desired passband response, say digital differentiator. 
ii) Magnitude zero constraint at the stopband:

Similarly, to impose $V_{\hat{\omega}_{s}}-1$ zeros on $H(z)$ at $\hat{\omega}_{s} \in \Omega$ (say in the stopband), we have

$$
\sum_{n=1}^{N-1} n^{v} \cdot h(n) \cdot e^{-j \hat{\omega}_{s} n}=0, v=0,1, \ldots, V_{\hat{\omega}_{s}}-1
$$

and (3-4) becomes:

$$
\boldsymbol{V}_{\hat{\omega}_{s}} \cdot \boldsymbol{h}=\boldsymbol{O}_{V_{\hat{\omega}_{s}}}, v=0,1, \ldots, V_{\hat{\omega}_{s}}-1 .
$$

where $\left[\boldsymbol{V}_{\hat{\omega}_{s}}\right]_{v, n}=n^{v} e^{-j \hat{\omega}_{s} n}$ and $\boldsymbol{O}_{V_{\hat{\omega}_{s}}}$ is a $V_{\hat{\omega}_{s}}$ null vector.

\section{B. - Removing redundant variables}

We now introduce a technique to impose the equality constraints structurally into the SDP formulation in section II-A. Suppose that there are $R$ different passband constraints with different degree of magnitude flatness $U_{\hat{\omega}_{p, r}}$ at $\hat{\omega}_{p, r} \in \Omega_{p}$ for $r=0,1, \ldots, R-1$, and $M$ different stopband constraints with different degree of magnitude zero $V_{\hat{\omega}_{s, m}}$ at $\hat{\omega}_{s, m} \in \Omega_{s}$ for $m=0,1, \ldots, M-1$, then according to (3-3) and (3-5), we have:

$\boldsymbol{U}_{\hat{\omega}_{p, r}} \cdot \boldsymbol{h}=\boldsymbol{d}_{\hat{\omega}_{p, r}} ; \boldsymbol{U}_{\hat{\omega}_{p, r}}$ and $\boldsymbol{d}_{\hat{\omega}_{p, r}}$ are $U_{\hat{\omega}_{p, r}} \times N$ matrix and

$U_{\hat{\omega}_{p, r}}$ column vector respectively, for $r=0,1, \ldots, R-1$; and

$\boldsymbol{V}_{\hat{\omega}_{s, m}} \cdot \boldsymbol{h}=\boldsymbol{O}_{{\hat{\hat{\omega}_{s}, m}}} ; \boldsymbol{V}_{\hat{\omega}_{s, m}}$ and $\boldsymbol{O}_{\hat{V}_{\hat{\omega}_{s, m}}}$ are $V_{\hat{\omega}_{s, m}} \times N$ matrix and

$V_{\hat{\omega}_{s, m}}$ column null vector respectively, for $m=0,1, \ldots, M-1$.

These constraints can be combined to form the following matrix representation of the linear constraints:

$\boldsymbol{A h}=\boldsymbol{b}$

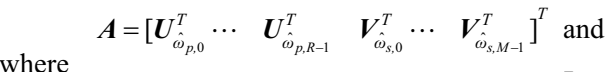

$$
\begin{aligned}
& \boldsymbol{b}=\left[\begin{array}{llllll}
\boldsymbol{d}_{\hat{\omega}_{p, 0}}^{T} \cdots & \boldsymbol{d}_{\hat{\omega}_{p, R-1}}^{T} & \boldsymbol{O}_{V_{\hat{\omega}_{s}, 0}}^{T} & \cdots & \boldsymbol{O}_{V_{\hat{\omega}_{s}, M-1}}^{T}
\end{array}\right]^{T} .
\end{aligned}
$$

Assume that the number of constraints is smaller than the number of variables, part of the variables, called the redundant variables, can be expressed in terms of the remaining variables, called the independent variables, when solving the SDP. First of all, rewrite (3-6) as follows:

$$
\left[\begin{array}{ll}
\boldsymbol{A}_{N-c} & \boldsymbol{A}_{c}
\end{array}\right] \cdot\left[\begin{array}{c}
\boldsymbol{h}_{N-c} \\
\boldsymbol{h}_{c}
\end{array}\right]=\boldsymbol{b},
$$

where $\boldsymbol{A}=\left[\begin{array}{ll}\boldsymbol{A}_{N-c} & \boldsymbol{A}_{c}\end{array}\right] ; \boldsymbol{h}=\left[\begin{array}{ll}\boldsymbol{h}_{N-c}^{T} & \boldsymbol{h}_{c}^{T}\end{array}\right]^{T}$; and $c$ is the number of redundant variables in $H(z)$. Using (3-7), $\boldsymbol{h}$ can be written in terms of $\boldsymbol{h}_{N-c}$ as:

$$
\boldsymbol{h}=\left[\begin{array}{c}
\boldsymbol{O}_{N-c} \\
\boldsymbol{A}_{c}^{-1} \boldsymbol{b}
\end{array}\right]+\left[\begin{array}{c}
\boldsymbol{I}_{N-c} \\
-\boldsymbol{A}_{c}^{-1} \boldsymbol{A}_{N-c}
\end{array}\right] \boldsymbol{h}_{N-c},
$$

where $\boldsymbol{I}_{N}$ is an $(N \times N)$ identity matrix; $\boldsymbol{O}_{N}$ is an $N$ column zero vector. By substituting (3-8) into (2-3), $\boldsymbol{h}_{N-c}$ can be found optimally by the SDP, while satisfying the prescribed constraints. Similarly, the above derivation can be readily applied to the complex FIR filter design by considering the real and imaginary parts of the linear equality constraints separately. Concerning the VDF design, linear constraints can be imposed on the VDF by imposing different constraints on each subfilter. More precisely, we have:

$$
\boldsymbol{A} \cdot \boldsymbol{h}_{l}=\boldsymbol{b}_{l} \text { for } l=0,1, \ldots, L-1,
$$

where

$$
\boldsymbol{b}_{0}=\boldsymbol{b} \text { and } \boldsymbol{b}_{l}=\left[\begin{array}{llllll}
\boldsymbol{O}_{U_{\hat{\omega}_{p}, 0}}^{T} & \cdots & \boldsymbol{O}_{U_{\hat{\omega}_{p}, R-1}}^{T} & \boldsymbol{O}_{V_{\hat{\omega}_{s, 0}}}^{T} & \cdots & \boldsymbol{O}_{V_{\hat{\omega}_{s, M-1}}^{T}}^{T}
\end{array}\right]^{T}
$$
for $l=1, \ldots, L-1$

Similarly, the redundant variables can be removed as in (3-8).

\section{C. - Peak error and convex quadratic constraints}

Apart from the linear equality constraints, linear and convex quadratic inequality constraints can easily be incorporated in the SDP formulation. As an illustration, we shall consider the optimal design of low-delay FIR filters with least squares stopband attenuation and prescribed peak ripple constraints. Let $\delta_{p}$ be the peak ripple to be imposed in a frequency band $\omega \in\left[\omega_{1}, \omega_{2}\right]$ (a collection of frequency bands is also feasible), then the peak error constraint can be written as:

$$
\left|H\left(e^{j \omega}\right)-H_{d}(\omega)\right|^{2} \leq \delta_{p}, \omega \in\left[\omega_{1}, \omega_{2}\right] .
$$

Similar to the minimax formulation, (3-10) can be rewritten as:

$$
\Lambda(\boldsymbol{h}, \omega)=\left[\begin{array}{ccc}
\delta_{p} & \beta_{R}(\omega) & \beta_{I}(\omega) \\
\beta_{R}(\omega) & 1 & 0 \\
\beta_{I}(\omega) & 0 & 1
\end{array}\right] \succeq 0, \quad \omega \in\left[\omega_{1}, \omega_{2}\right],
$$

where $\beta_{R}(\omega)=\left|\boldsymbol{h}^{T} \boldsymbol{c}(\omega)-H_{R_{-} d}(\omega)\right|$ and $\beta_{I}(\omega)=\left|\boldsymbol{h}^{T} \boldsymbol{s}(\omega)+H_{I_{-} d}(\omega)\right|$. Digitizing (3-11), the resulting constraints on the peak ripples can be augmented to the existing LMI in (2-11) and (2-14) for the minimax and LS criterion respectively. Peak error constraints for complex FIR filters and VDFs can be derived similarly, using the definitions in (2-15) and (2-21) respectively.

\section{Design EXamples}

\section{Example 1: Low-delay Hilbert Transformers (HTs)}

In this example, low-delay HTs with prescribed magnitude flatness are designed. Due to space limitation, only the minimax design of real-valued odd-length HTs will be given. The desired frequency response of the HT $[8,14]$ is given by:

$$
H_{d}^{H T}(\omega)=-j e^{-j \omega \tau} \quad \omega_{p 1}<\omega<\omega_{p 2},
$$

where $\omega_{p 1}$ and $\omega_{p 2}$ are the passband cutoff frequencies and $\tau=(N-1) / 2-D$ is the group delay. It should be noted that when the reduction parameter $D$ is equal to zero, the proposed HTs with odd- and even-lengths correspond to the traditional type-3 and type-4 linear-phase HTs respectively. As an illustration, $D$ is set to 2 in this example. Substituting (4-1) into (3-2), a set of magnitude flatness constraints for HTs can be readily derived. The filter length $N$ for the odd-length HTs, is 21 and the passband is from $0.2 \pi$ to $0.8 \pi$. Figure 1 shows the frequency and group delay responses of the odd-length HTs with $U_{0.5 \pi}=0$ and 3. It can be seen that the passband ripples are smaller for the designs with lower order of magnitude flatness, which is to be expected. Moreover, unlike the linear-phase HTs, the proposed low-delay HTs do not necessarily have a zero magnitude at $\omega=0$ and $\pi$. As a comparison, the conventional type-3 maximally-flat linear-phase HT (MF-LP-HT) [14] is also designed and plotted as dotted line in figure 1 . The filter length of the type-3 MF-LP-HT is chosen to be 17 so that it has the same group delay as the proposed low-delay HTs. It is noticed that the passband errors of the type-3 MF-LP-HT increase as the frequencies go further away from $\omega=\pi / 2$. For the current design, the passband error of the type-3 MF-LP-HT at $\omega=0.2 \pi$ or $0.8 \pi$ is $0.6728 \mathrm{~dB}$, which are significantly larger than the worst-case passband errors $(0.006285 \mathrm{~dB})$ of the proposed design with second order magnitude flatness. In order to decrease the errors at the required passband cutoff frequencies, the length and hence the system delay of the type-3 MF-LP-HT has to be increased significantly. This suggests that the prescribed flatness approach offers more flexibility and design freedom in satisfying different passband and stopband requirements than the conventional method.

\section{Example 2: Low-delay complex single passband filters}

In this example, low-delay complex single passband filters of length $N=31$ with magnitude flatness and zero are designed. The constraint parameters, as discussed in Section III-A, are $U_{0}=3$ and $V_{\pi}=3$. Both minimax and least-squares stopband criteria, with equal passband and stopband weights, are considered. The specifications of the filters are: 


$$
H_{d}(\omega)=\left\{\begin{array}{ll}
e^{-j 12 \omega}-0.1 \pi<\omega<0.3 \pi \\
0 & -\pi<\omega<-0.2 \pi \text { and } 0.4 \pi<\omega<\pi
\end{array} .\right.
$$

The frequency and group delay responses of the filters so obtained are plotted in figure 2. The worst-case stopband attenuation at $\omega \in[0.4 \pi, \pi]$ is $23.77 \mathrm{~dB}$ for the LS design, as compared to $30.25 \mathrm{~dB}$ for the minimax design. To further illustrate the flexibility of the SDP method, peak error constraints, which are convex quadratic inequality constraints, are imposed to limit the sidelobe at $\omega \in[0.4 \pi, 0.6 \pi]$ to $29 \mathrm{~dB}$ in exchange for slightly lower performance at the unconstrained frequency bands. From figure 2 and the pole-zero plot (not shown here due to page limitation), it is noticed that both equality and inequality constraints are satisfied.

\section{Example 3: Low-delay tunable lowpass filter (VDF)}

In this example, low-delay FIR lowpass filter with variable cutoff frequency is designed using the proposed SDP formulation. The transition bandwidth is fixed at $0.2 \pi$ and the passband edge is varied from $0.2 \pi$ to $0.4 \pi$. The FIR VDF has a subfilter length of 30 , an interpolation order of 5 using the polynomial basis function, and a constant group delay of 12.5 in the passband. First order magnitude flatness at $\omega=0$ and first order magnitude zero at $\omega=\pi$ are enforced in our VDF design: i.e. $U_{0}=2$ and $V_{\pi}=2$. Figure $3 \mathrm{a}$ shows the frequency response with evenly sampled tuning parameters $\phi \in[0,1]$ and figure $3 b$ shows the group delay response of the lowpass FIR VDF so obtained using minimax criterion. The corresponding passband deviation and stopband attenuation are respectively $0.0398 \mathrm{~dB}$ and $46.678 \mathrm{~dB}$. Alternatively, we can solve the above problem in LS sense. However, the design results using LS criterion with flatness and/or peak error constraints are omitted due to page limitation. It should be noted that such constrained design problem has not been studied in conventional design methods of VDFs. Also the VDFs considered in [15] are based on the LS criterion without any additional constraints.

\section{Conclusion}

An SDP approach for designing real and complex FIR filters and VDF with prescribed flatness and peak error constraints has been presented. These constraints can be easily incorporated in the SDP formulation to obtain optimal passband linear-phase FIR filters with minimax or LS design criteria. In addition, a technique for structurally imposing the equality constraints in the SDP is proposed. Design examples are given to illustrate the effectiveness of the proposed approach. It shows that the SDP method is an attractive alternative to traditional design methods in tackling a wide range of filter design problems, because of its optimality, generality and flexibility.

\section{REFERENCES}

[1] W.-S Lu, "A unified approach for the design of 2-D digital filters via semidefinite programming", IEEE Trans. Circuits Syst. I, vol. 49, no. 6, pp. 814-826, Jun 2002.

[2] W.-S Lu, "Design of nonlinear-phase FIR digital filters: A semidefinite programming approach", ISCAS'99, vol. III, pp. 263266, Orlando, FL, May 1999.

[3] S.-P Wu, S. Boyd, and L. Vandenberghe, "FIR filter design via semidefinite programming and spectral factorization", Proc. 5th Conf. Decision and Control,pp. 271-276, Dec. 1996.

[4] W.-S Lu, "Design of stable minimax IIR digital filters using semidefinite programming," in Proc. ISCAS'2000, pp. I-355-358, Geneva, Switzerland, May 28-31, 2000

[5] K. S. Pun and S. C. Chan, "The Minimax Design of Digital Allpass Filters with Prescribed Pole Radius Constraint Using Semidefinite Programming (SDP)," in Proc. ICASSP'2003, vol. 6, pp. 413-416, Hong Kong.

[6] W.-S Lu and A. Antoniou, "Design of Digital Filters and Filter Banks by Optimization: A state of the Art Review," in Proc. EUSIPCO'2000, vol. 1, pp. 351-354, Sep. 2000.
[7] H. Wolkowicz, R. Saigal and L. Vandenberge, "Handbook of Semidefinite Programming: theory, algorithms, and applications", Kluwer Academic Publishers, 2000.

[8] S. K. Mitra and J. F. Kaiser, "Handbook for Digital Signal Processing", John Wiley \& Sons, Inc., 1993.

[9] S. C. Pei, C. C. Tseng and W. S. Yang, "FIR Filter Designs with Linear Constraints Using the Eigenfilter Approach," IEEE Trans. Circuits Syst. II, Vol. 45, pp. 232-237, No. 2, Feb, 1998.

[10] Y. Wisutmethangoon and T. Q. Nguyen, "A Method for Design of $M$ th-Band Filters," IEEE Trans. Signal Processing, vol. 47, no. 6, pp. 1669-1678, June, 1999.

[11] S. C. Pei and J. J. Shyu, "Complex Eigenfilter Design of Arbitary Complex Coefficient FIR Digital Filters", IEEE Trans. Circuits Syst. II, Vol. 40, No. 2, pp. 32-40, Jan, 1993.

[12] T. Q. Nguyen, "The Design of Arbitrary FIR Digital Filters Using the Eigenfilter Method," IEEE Trans. Signal Processing, vol. 41, no. 3, pp. 1128-1139, March, 1993.

[13] J. W. Adams, "FIR Digital Filters with Least-Squares Stopbands Subject to Pain-Gain Constraints", IEEE Trans. Circuits Syst. vol. 39, no 4, pp. 376-388, Nov, 1991.

[14] T. Cooklev and A. Nishihara, "Maximally Flat FIR Hilbert Transformers," Int. J. Circuit Theory Appli., vol. 20, no. 6, pp. 563-570, 1993.

[15] K. S. Pun, S. C. Chan, K. S. Yeung and K. L. Ho, "On the Design of FIR and IIR Digital Filters with Variable Frequency Characteristics," IEEE Trans. Circuits Syst. II, vol. 49, no. 11, pp. 689-703, Nov, 2002.

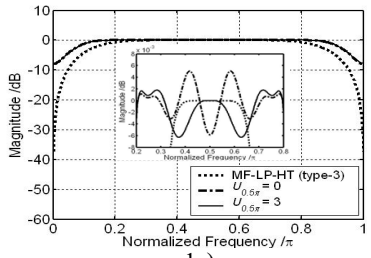

1a)

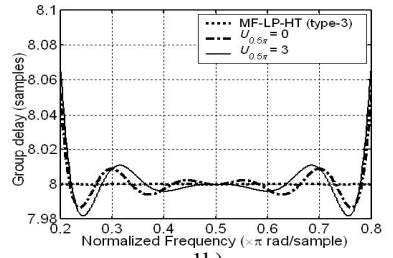

1b)
Figure 1: Design results of low-delay odd-length HTs in example 1 (Design Criterion: Minimax): a) Frequency response (Passband details in smaller figure). b) Group delay response. (MF-LP-HT: Maximally-flat Linear-phase Hilbert transformer).

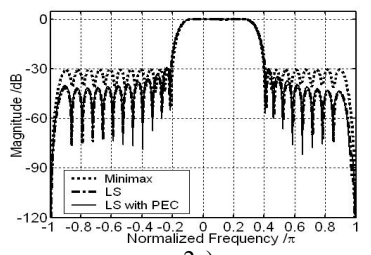

2a)

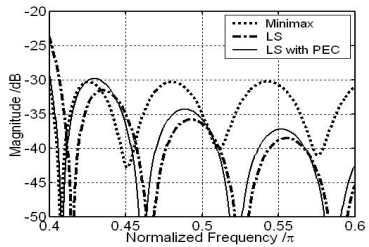

2c)

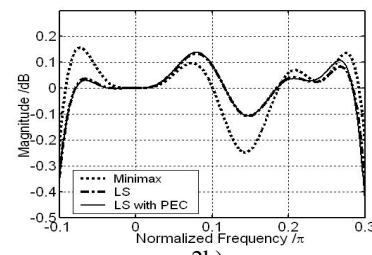

2b)

2d)

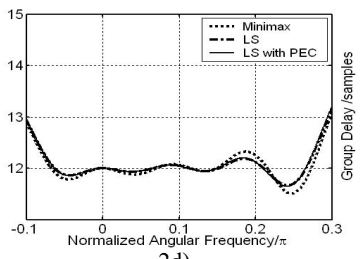

Figure 2. Design results of low-delay complex single passband filters in example 2 (Linear equality constraint: $U_{0}=3$ and $V_{\pi}=3$ ): a) Frequency response. b) Passband details c) Stopband details at $\omega \in[0.4 \pi, 0.6 \pi]$. d) Group delay response. (PEC: $-29 \mathrm{~dB}$ Peak Error Constraint at $\omega \in[0.4 \pi, 0.6 \pi]$ ).

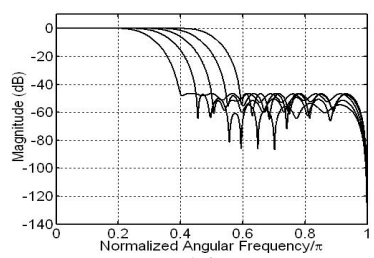

3a)

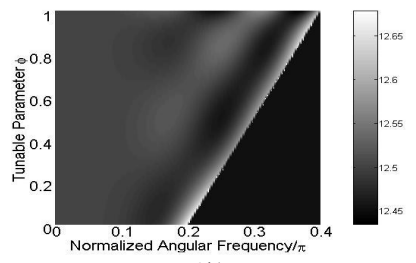

3b)
Figure 3: Design results of low-delay VDF in example 3 (Linear equality constraint: $U_{0}=2$ and $V_{\pi}=2$. Design Criterion: Minimax): a) Frequency response with $\phi$ evenly sampled in the range [0,1] . b) Group delay response. 